\title{
Estrés oxidativo, prooxidantes y enfermedad de Crohn
}

\author{
Alexis Tapia Sa, Magdalena Araya M. \\ Oxidative stress, prooxidants and \\ Crohn disease
}

The frequency of Crohn disease shows a significant increase in the last century. Affected patients show a misbalance between production of free radicals and anti oxidant defenses. Thus, one of the increasingly accepted htypotheses to explain the origin of this disease, is the oxidative stress that occurs in the intestinal mucosa. Dietary factors including self administered vitamin and mineral supplemets may play a role, especially when they contain excessive amounts of iron and copper, known for their prooxidant capacities. Unfortunately, little is known about how these metals may influence the antioxidant defenses in the intestinal mucosa. This article reviews the literature on the why and how these elements may act on individuals susceptible to develop Crohm disease, including the evidence supporting the hypothesis that oxidative stress in the intestinal mucosa is an important pathogenetic factor (Rev Méd Chile 2006; 134: 95-100).

(Key-words: Crohn disease; Inflammatory bowel diseases; Oxidative stress; Reactive oxygen species)

Recibido el 14 de septiembre, 2004. Aceptado el 20 de abril, 2005.

Instituto de Nutrición y Tecnología de los Alimentos (INTA)

aAlumno programa de Doctorado en Nutrición y Alimentos. Universidad de Chile.

$\mathrm{E}^{\mathrm{n}}$ las últimas décadas se ha tomado conciencia sobre la magnitud del cambio del patrón epidemiológico de enfermedades a escala mundial. Una de las hipótesis más aceptadas, postula que modificaciones en la interacción del hombre con el ambiente serían responsables de estas variaciones ${ }^{1}$; en este contexto, una argumentación muy sólida se relaciona a la higiene y alteraciones en el entorno microbiológico, tanto del ambiente como de la flora intestinal residente, que determinarían cambios sustanciales en la manera de reaccionar del sistema inmune.

Correspondencia a: Magdalena Araya M. Instituto de Nutrición y Tecnología de los Alimentos (INTA), Universidad de Chile. Macul 5540, Casilla 13811, Santiago, 11, Chile. Teléfono: 678 1468. Fax: 221 4030. E-mail: maraya@inta.cl
La enfermedad de Crohn es una de las condiciones que ha aumentado significativamente durante el último siglo ${ }^{2}$. Actualmente, la incidencia en Estados Unidos se estima en 5 por 100.000 habitantes $^{3}$ y en Inglaterra, en 1 por 1.500 habitantes $^{4}$. La etiología permanece desconocida; la susceptibilidad genética y la participación del sistema inmune son ampliamente aceptadas como factores participantes en su patogenia, en cambio, el rol de la dieta, que representa otro factor participante, está poco claro. El estrés oxidativo en la mucosa ha ido cobrando relevancia progresiva entre las hipótesis que buscan explicar la patogenia, ya que en pacientes afectados por esta enfermedad se ha encontrado un desequilibrio entre la producción de radicales libres y defensas antioxidantes. En este punto, la dieta y sus componentes con capacidad 
prooxidante, como por ejemplo el hierro y algunos microminerales, como el cobre, merecen ser considerados. Tomando en cuenta la creciente frecuencia de exposición a minerales que existe en la población a través de la autoadministración de diversos suplementos, hemos considerado oportuno revisar la literatura acerca de por qué y cómo estos elementos pueden ser deletéreos a través de su efecto prooxidante para las personas susceptibles de desarrollar enfermedad de Crohn.

\section{Minerales y microminerales}

El hierro es uno de los metales más importante en los tejidos de los mamíferos, en el hombre oscila entre 3 y $4 \mathrm{~g}$. Los llamados microminerales están presentes en menor cantidad, por ejemplo, en nuestros tejidos existen aproximadamente $80 \mathrm{mg}$ de cobre. En el hombre, dos terceras partes del hierro están contenidas en la hemoglobina; la mioglobina contribuye con $300 \mathrm{mg}$ y el resto de enzimas que portan hierro suman unos $150 \mathrm{mg}$ (4\%-5\% del total). Entre los microminerales, el cobre también forma parte de enzimas y proteínas indispensables para la vida, como citocromo C oxidasa, ferroxidasa, tirosinasa, dopamina beta hidroxilasa, lisil oxidasa y cobre-zinc superoxidasa dismutasa (Cu, $\mathrm{Zn}-\mathrm{SOD})$. Por lo tanto, la deficiencia de estas enzimas debido a la carencia de cobre, lleva a deterioro de funciones tan relevantes como la respiración celular, oxidación de hierro, formación de pigmentos, biosíntesis de neurotransmisores, formación de tejido conectivo y defensa antioxidante ${ }^{5}$.

Ambos metales se absorben en el intestino delgado. La dieta normal contiene 13-18 mg de hierro al día, de los cuales sólo un $1 \mathrm{mg}$ es absorbido $^{6}$. El cuerpo humano regula la homeostasis del hierro controlando la absorción, pues no existe excreción de hierro en condiciones fisiológicas ${ }^{7}$, sino que se pierde a través de células descamadas de las vías digestiva, urinaria y aérea, estimándose en una cantidad de $14 \mu \mathrm{g} / \mathrm{kg}$ peso corporal/ día ${ }^{8}$. A su vez, la absorción se relaciona inversamente con los depósitos de hierro y directamente con la velocidad de la eritropoyesis. Es decir, la absorción puede aumentar de acuerdo a los requerimientos metabólicos, de lo que se desprende que estos serán distintos considerando sexo y etapa del desarrollo. Por lo tanto, la dosis diaria recomendada también varía de acuerdo a la edad, sexo y biodisponibilidad del hierro en la dieta. La dosis más alta tolerable, en Estados Unidos, es de $45 \mathrm{mg} /$ día, nivel basado en la aparición de manifestaciones gastrointestinales como constipación, náuseas, vómitos o diarrea.

En cuanto a cobre, aproximadamente la mitad de su ingesta diaria, 1- 2 mg, es absorbida9, cantidad parecida a la de hierro, pero debemos recordar que en el cuerpo humano hay hasta 50 veces más hierro que cobre. Los alimentos más ricos en este metal son las legumbres, carne de vacuno y mariscos; habitualmente, el agua de bebida no representa mas de $10 \%$ de la ingesta diaria. Actualmente, la OMS ha definido una concentración de $2 \mathrm{mg}$ de cobre por litro de agua como una concentración segura para la salud humana. Las molestias gastrointestinales al ingerir agua con cobre en concentraciones mayores comienzan a aparecer a los $3 \mathrm{mg} \mathrm{Cu} / \mathrm{L}^{10}$. Los signos y síntomas por envenenamiento son gusto metálico, náusea, diarrea, ictericia, hemoglobinuria, hematuria, anuria y oliguria ${ }^{11}$. En las condiciones ácidas del estómago, los iones $\mathrm{Cu}$ son liberados de los alimentos en los que vienen unidos; estos iones son rápidamente atrapados por aminoácidos, como histidina, treonina, glutamina, ácidos orgánicos y otras moléculas con capacidad quelante. Aunque se puede absorber cobre desde la mucosa gástrica, la mayor parte de este proceso ocurre en el intestino delgado. Se ha demostrado que el porcentaje de absorción es variable, dependiendo de la carga dietaria; cuando hay una exposición a altas concentraciones de cobre, se absorbe un menor porcentaje (12\%). Por el contrario, cuando la ingesta es escasa, el porcentaje de absorción aumenta $(60 \%)^{12}$.

Estudios en células Caco 2, sugieren que el tránsito de cobre varía según la célula esté o no cargada previamente con el elemento ${ }^{13,14}$. El transporte plasmático del cobre está a cargo, principalmente, de la ceruloplasmina y una fracción circula unida a una serie de proteinas más pequeñas, entre las que destaca la albúmina, por su sitio de unión de alta afinidad para $\mathrm{Cu}^{+2}$ en su cola amino terminal ${ }^{15}$. Otra proteína involucrada es la transcupreina, actualmente no totalmente caracterizada, que tendría importancia principalmente en el transporte desde el intestino al hígado. La histidina es otro elemento que participa del transporte del cobre y se le postula como una de las moléculas que puede entregar cobre a las células. 
Toxicidad de hierro y cobre es mediada por estrés oxidativo:

El estrés oxidativo es una condición que se presenta cuando hay un desbalance entre la producción de especies reactivas de oxígeno (ERO) potencialmente tóxicas, tales como el anión superóxido $\left(\mathrm{O}_{2}{ }^{-0}\right)$, peróxido de hidrógeno $\left(\mathrm{H}_{2} \mathrm{O}_{2}\right)$ y el radical hidroxilo $\left(\mathrm{OH}^{-0}\right)$ y las actividades de los sistemas de secuestro, en los que se incluyen enzimas (superóxido dismutasa, catalasa, peroxidasa y peroxiredoxina), especies antioxidantes de bajo peso molecular (vitamina E, ascorbato, glutatión), además de otras complejas formas de protección como transportadores de metales e inducción de factores de transcripción.

Los iones Fe y $\mathrm{Cu}$ tienen la capacidad de donar y aceptar electrones, lo que les permite reaccionar con otros compuestos generando ERO, las que pueden afectar rápidamente a las membranas celulares, proteínas y $\mathrm{ADN}^{16}$. Por esta razón, los sistemas biológicos propenden a que estos metales no se encuentren libres, sino que unidos a proteínas, tanto para su transporte como para su almacenamiento. Es así que el hierro libre $\mathrm{Fe}^{2+}$ es inmediatamente quelado en las células por componentes tales como citrato o adenosin difosfato. Sin embargo, el «hierro lábil» puede participar en la formación del radical hidróxilo $\mathrm{OH}^{-0}$ (reacción de Haber-Weiss). Por su parte, este radical, cuya corta vida media se estima en 10-9 segundos, puede dañar lípidos, proteínas, ADN, azúcares, y generalmente, todas las moléculas orgánicas ${ }^{17}$.

Por su capacidad de inducir daño en múltiples objetivos a nivel intracelular, el estrés oxidativo ha sido involucrado en una variedad de patologías. Entre las patologías con las que se relaciona se incluyen las infecciosas, atribuyéndosele roles en la alta mortalidad del shock endotóxico ${ }^{18}$, en la patogénesis de enfermedades gástricas por Helicobacter pylori, incluyendo inflamación, ulceración y carcinogénesis ${ }^{19}$, y como factor contribuyente al desarrollo de la malaria ${ }^{20}$. Se considera mediador del daño vascular de enfermedades cardiovasculares como hipertensión, ateroesclerosis e isquemiareperfusión ${ }^{21}$. Su presencia se asocia también con varios tumores, como próstata ${ }^{22}$, cuello uterino ${ }^{23}$, ovario $^{24}$ y de hígado 25 .

En cuanto a hierro y estrés oxidativo, existen estudios que han demostrado que marcadores de estrés oxidativo como sustancias reactivas con ácido tiobarbitúrico (TBARS), que reflejan la peroxidación lipídica, son significativamente más altos en pacientes con hemocromatosis. Además, en estos pacientes se encuentran reducidos los niveles de un secuestrador de radicales libres como es el glutation. Concordantemente, se ha reportado que las TBARS disminuyen significativamente con la terapia de depleción de hierro ${ }^{26}$. En animales, una sobrecarga de hierro resulta en peroxidación lipídica hepática ${ }^{27}$, oxidación de proteínas hepáticas ${ }^{28} \mathrm{y}$ daño oxidativo asociado a otros tejidos, incluidos los eritrocitos ${ }^{29}$. Por otro lado, el fierro y las ERO participarían en procesos neurodegenerativos como la enfermedad de Alzheimer, esclerosis lateral amiotrófica, enfermedad por priones y de Parkinson ${ }^{30}$.

En lo referente a daño por cobre, la exposición a un exceso del metal tiene dos tipos de efectos: los resultantes de su acumulación en el hígado, que lleva eventualmente a daño hepático grave, cirrosis e insuficiencia hepática ${ }^{31}$, y los resultantes de su depósito y acción sobre el sistema nervioso central. Por ejemplo, su presencia se asocia a la neurotoxicidad del péptido $\beta$ amiloide en la enfermedad de Alzheimer, produciéndose daño neuronal mediado por estrés oxidativo ${ }^{32}$. Desgraciadamente, aunque el cobre tiene capacidad prooxidante, no se ha demostrado la relación entre cobre y la aparición de daño o enfermedad.

Hierro, cobre y enfermedad de Crohn:

Durante la digestión, el hierro y cobre que son liberados de los alimentos pueden existir como iones en el tubo digestivo y participar en la generación de radicales libres. De los 13-18 mg de Fe ingeridos al día, sólo $1 \mathrm{mg}$ es absorbido, lo que ocasiona que alrededor de $15 \mathrm{mg}$ del metal puedan seguir su camino por el lumen intestinal, tanto delgado como grueso y ejercer un efecto prooxidante sobre las células de la mucosa. La absorción del cobre presenta un fenómeno parecido.

Aunque no existe evidencia que demuestre que el estrés oxidativo cause una enfermedad determinada, desde hace años existen antecedentes de que el daño oxidativo inducido por hiemo en el tubo digestivo, podnía estar relacionado con procesos como aparición de tumores ${ }^{33}$. También se ha asociado a un efecto negativo en enfermedades inflamatorias intestinales crónicas, por ejemplo la enfermedad de Crohn, en la que se postula que su presencia exacerbana la 
sintomatología. La enfermedad de Crohn puede afectar a cualquier parte del tubo digestivo, desde la boca hasta el ano. Se manifiesta principalmente como dolor abdominal, diarrea, baja de peso y ocasionalmente como masa abdominal, obstrucción intestinal o fístula. La etiología de esta enfermedad aún no es entendida completamente, sin embargo, se atribuye cierta participación a factores ambientales, inmunológicos y genéticos ${ }^{34}$. En la patogenia se sabe que la inflamación intestinal se acompaña de una producción excesiva de especies reactivas de oxígeno y metabolitos de nitrógeno, pero no está claro si la participación de estas ERO estańan asociadas a la patogenia de la enfermedad o es un factor interviniente que modulanian su evolución. Para contramestar los efectos deletéreos de los radicales libres, la mucosa intestinal ejerce la defensa antioxidante en una vía de dos pasos: en primer lugar la enzima superóxido dismutasa (SOD) convierte el $\mathrm{O}_{2}^{-0}$ a $\mathrm{H}_{2} \mathrm{O}_{2}$, el que en segundo lugar es neutralizado a $\mathrm{H}_{2} \mathrm{O}$ por las enzimas catalasa (CAT) o glutatión peroxidasa (GPO-px). Se ha demostrado que los niveles de los más importantes antioxidantes se encuentran disminuidos en la mucosa intestinal de pacientes con enfermedades inflamatorias intestinales 35 .

Por otro lado, un estudio en que a pacientes con enfermedad de Crohn se les trató la anemia con 120 $\mathrm{mg} /$ día de fumarato ferroso, se demostró un agravamiento de la diarrea, dolor abdominal y náuseas, lo que correlacionó con una disminución significativa en plasma de cisteína y glutatión reducido (GSH). Esta evidencia apoya el hecho de que el estrés oxidativo media la patogenia de la enfermedad, ya que el hierro administrado deterioró el estado antioxidante del plasma y aumentó la sintomatología de los pacientes ${ }^{36}$. Aunque no existe evidencia para el cobre, podríamos postular que este puede tener efectos similares. Si consideramos que la mayonía de los pacientes con enfermedad inflamatoria intestinal presentan anemia por deficiencia de hierro en alguna etapa de su enfermedad, la necesidad de tratarlos con fierro por vía oral es frecuente ${ }^{37}$. En sujetos sanos, a quienes se dio una suplementación de $19 \mathrm{mg}$ de fierro elemental al día por un período de dos semanas, se observó que los radicales libres en las deposiciones aumentaron alrededor de $40 \% 38$. Así, la evidencia indica que el hierro produce estrés oxidativo intraluminal, pero no aclara si el fenómeno tiene una magnitud que puede ser manejada por los mecanismos homeostáticos que habitualmente ope- ran en el intestino al entrar en contacto con la carga dietaria o si constituyen un riesgo per se.

En otro estudio, se suplementó a pacientes con enfermedad de Crohn inactiva o medianamente activa con vitaminas E (800 UI) y C (1000 mg), ambas conocidas por su capacidad antioxidante, durante cuatro semanas, tiempo en el cual disminuyeron los indicadores plasmáticos de estrés oxidativo F2-isoprostanos 39 . En este tipo de pacientes también se ha demostrado una disminución significativa de vitaminas antioxidantes plasmáticas como ácido ascórbico, alfa y beta carotenos, licopenos y betacriptoxantina ${ }^{40}$.

En modelos animales existe evidencia del papel del fierro y del estrés oxidativo en el empeoramiento del daño en la mucosa intestinal inflamada. En ratas a las que se les induce químicamente colitis, y se les da a ingerir fierro, se demostró un aumento de la actividad de la enfermedad, las que disminuyeron al adicionarles el efecto antioxidante de la vitamina $\mathrm{E}^{41}$. Además el aumento de la actividad de la enfermedad se asoció con un aumento del estrés oxidativo ${ }^{42}$. En ratas a las que solamente se indujo colitis, sin administrarles hierro, pero sí se les suplementó vitamina E, se observó una disminución en la severidad de las lesiones colónicas ${ }^{43}$. En forma parecida se demostró que al inyectarles glutatión antes de inducir la colitis mejoró significativamente el daño colónico, se restauraron los niveles de GSH y cisteína, y disminuyó la peroxidación de lípidos ${ }^{44}$.

Hasta ahora no existen datos sobe el papel del cobre en el estrés oxidativo del tubo digestivo humano. Es importante que se realicen estos estudios, dado el aumento del uso de suplementos de cobre y de las propuestas, que ya se discuten por algunos grupos, de suplementarlo en individuos considerados vulnerables, como aquellos con osteoporosis o riesgo cardiovascular.

La evidencia analizada demuestra que los pacientes portadores de la enfermedad de Crohn, ya sea activa o inactiva, presentan un desbalance en detrimento de defensas antioxidantes y un aumento de las especies reactivas de oxígeno y radicales libres. Como ya se mencionó, la etiología de la enfermedad es desconocida y su incidencia está aumentando. Dadas las implicaciones del estrés oxidativo en su patogenia, es posible especular que parte de este aumento puede estar relacionado a la mayor frecuencia de dietas desbalanceadas en la 
población, que tienden a ser deficientes en vitaminas antioxidantes y contener metales prooxidantes (como cobre y hierro) en exceso ${ }^{45}$. Ante el potencial riesgo que representa la presencia de iones libres de hierro y cobre en el intestino delgado, resulta clara la necesidad de realizar estudios. Es crucial comprender cómo la mucosa intestinal maneja el estrés oxidativo producido por estos metales, ya sea en dosis fisiológicas o suprafisiológicas. Sería interesante analizar desde esta perspectiva los programas de fortificación y suplementación de hierro, a través de los cuales individuos, tanto deficientes como no deficientes

\section{REFERENCIAS}

1. BACH JF. Infections and susceptibility to autoimmune and allergic disease. N EngJ Med 2002; 347: 911-20.

2. Bengmark S. Ecological control of the gastrointestinal tract. The role of probiotic flora. Gut 1998; 42: 2-7.

3. Lichtenstein G. Reduction of Colorectal Cancer Risk in Patients with Crohn's Disease. Rev Gastroenterol Disord 2002; 2: 16-24.

4. RAmpton D. Management of Crohn's disease. BMJ 1999; 319: 1480-5.

5. Cox D, Moore S. Copper Transporting P-Type ATPases and HumanDisease. J Bioenerg Biomembr 2002; 34: 333-8.

6. MiRET S, Simpson R. Physiology and molecular biology of dietary iron absortion. Ann Rev Nutr 2003; 23: 283-301.

7. ANDREWs N. Disorders of iron metabolism. NEngl J Med 1999; 341: 1986-95.

8. GREEN R. Body iron excretion in man. A colloborative study. Am J Med 1968; 45: 336-53.

9. Linder M, Wooten L, Cerveza P, Cotton S, Shuzze R, LOMEL N. Copper transport. Am J Clin Nutr 1998; 67: 965-71.

10. FItZgerald D. Safety guidelines for copper in water. Am J Clin Nutr 1998; 67: 1098-102.

11. BREMNER I. Manifestations of copper excess. Am J Clin Nutr 1998; 67: 1069-73.

12. Turnlund J, Keyes W, Anderson H, Acord L. Copper absorption and retention in young men at three levels of dietary copper using the stable isotope 65Cu. Am J Clin Nutr 1989; 49: 870-8. en hierro, reciben este elemento. Estos programas no consideran la presencia de polimorfismos presentes en la población, relacionados al metabolismo de metales y manejo de antioxidantes, que pueden significar que existen grupos de individuos vulnerables con mayor riesgo de desarrollar enfermedad de Crohn al estar expuestos a una carga oxidativa crónica. No existe evidencia publicada sobre el potencial papel del cobre en la enfermedad de Crohn. Sin embargo, parece razonable plantear que debemos establecer las dosis de ingesta segura de metales con capacidad prooxidativa para proteger adecuadamente a la población.

13. ARREDondo M, UauY R, GonzÁlez M. Regulation of copper uptake and transport in intestinal cell monolayers by acute and chronic copper exposure. Biochim Biophys Acta 2000; 1474: 169-76.

14. Levenson $C$, JaNGHoRBAN M. Long-term measurement of organ copper tumover in rats by continuous feeding of a stable isotope. Anal Biochem 1994; 221: 243-9.

15. LEVENSON C. Mechanisms of copper conservation in organs. Am J Clin Nutr 1998; 67: 978-81.

16. Gutteridge J, Rowley D, Hawwell B. Superoxidedependent formation of hydroxyl radical and lipid peroxidation in the presence of iron salt: detection of catalytic iron and antioxidant activity in extracellular fluids. Biochem J 1982; 206: 605-9.

17. Nelson S, McCord J. Iron, oxygen radicals, and disease. Adv Mol Cell Biol 1998; 25: 157-83.

18. Victor V, Rocha M, De La Fuente M. Immune cells: free radicals and antioxidants in sepsis. Int Immunopharmacol 2004; 4: 327-47.

19. Baek HY, Lim JW, Kim H, Kim JM, Kim JS, Jung HC ET AL. Oxidative-stress-related proteome changes in Helicobacter pylori-infected human gastric mucosa. Biochem J 2004; 379: 291-9.

20. Becker K, Tiley L, Vennerstrom JL, Roberts D, Rogerson S, GinsBurg H. Oxidative stress in malaria parasite-infected erythrocytes: host-parasite interactions. Int J Parasitol 2004; 34: 163-89.

21. Touyz R. Reactive oxygen species in vascular biology: role in arterial hypertension. Expert Rev Cardiovasc Ther 2003; 1: 91-106.

22. SiKKA S. Role of oxidative stress response elements and antioxidants in prostate cancer pathobiology and chemoprevention-a mechanistic approach. Curr Med Chem 2003; 10: 2679-92. 
23. Manju V, Balasubramanian V, Nalni N. Oxidative stress and tumor markers in cervical cancer patients. J Biochem Mol Biol Biophys 2002; 6: 387-90.

24. Senthil K, Aranganathan S, Nalini N. Evidence of oxidative stress in the circulation of ovarian cancer patients. Clin Chim Acta 2004; 339: 27-32.

25. Lu D, Peng Z, Qiu G, Zhou C. Expression of telomerase activity and oxidative stress in human hepatocellular carcinoma with cirrhosis. World J Gastroenterol 2003; 9: 1859-62.

26. Gaenzer H, Marschang P, Sturm W, Neumayr G, Vogel W, Patsch J et al. Association between increased iron stores and impaired endothelial function in patients with hereditary hemochromatosis. J Am Coll Cardiol 2002; 40: 2189-94.

27. Britton R, Ferrali M, Magiera C, Recknagel R, BACON B. Increased prooxidant action of hepatic cytosolic low-molecular-weight iron in experimental iron overload. Hepatology 1990; 11: 103843.

28. Galdeano M, Puntarulo S. Dietary alpha-tocopherol supplementation on antioxidant defenses after in vivo iron overload in rats. Toxicology 1997; 124: 73-81.

29. Galizano M, Puntarulo S. Role of antioxidants on the erythrocytes resistance to lipid peroxidation after acute iron overload in rats. Biochim Biophys Acta 1995; 1271: 321-6.

30. Thompson K, Shoham S, Connor J. Imon and neurodegenerative disorders. Brain Res Bull 2001; 55: 155-64.

31. O’Donohue J, Reid M, Varghese A, Portmann B, Wiwams R. Micronodular cirrhosis and acute liver failure due to chronic copper self-intoxication. Eur J Gastroenterol Hepatol 1993; 5: 561-2.

32. Dikalov SI, Vitek MP, Mason RP. Cupric-amyloid beta peptide complex stimulates oxidation of ascorbate and generation of hydroxyl radical. Free Radic Biol Med 2004; 36:340-7.

33. TоYokUNI S. Iron-induced carcinogenesis: the role of redox regulation. Free Radic Biol Med 1996; 20: 553-66.

34. Xia B, Crusius J, Meuwissen S, Pena A. Inflammatory bowel disease: definition, epidemiology, etiologic aspects, and immunogenetic studies. World J Gastroenterol 1998; 4: 446-58.

35. Kruidenier L, Kuiper I, Lamers CB, Verspaget HW. Intestinal oxidative damage in inflammatory bowel disease: semi-quantification, localization, and association with mucosal antioxidants. J Pathol 2003; 201: 28-36.

36. ERichsen K, Hausken T, Ulvik RJ, Suardal A, Berstad A, BERGE RK. Ferrous fumarate deteriorated plasma antioxidant status in patients with $\mathrm{Crohn}$ disease. Scand J Gastroenterol 2003; 38: 543-8.

37. Oldenburg B, Koningsberger J, Van Berge HenegOUWEN G, VAN AsBeck B, MarX M. Iron and iflammatory bowel disease. Aliment Pharmacol Ther 2001; 15: 429-38.

38. Lund E, Wharf S, Fairmeather-Tait J, Johnson I. Oral ferrous sulfate supplements increase the free radical-generating capacity of feces from healthy volunteers. Am J Clin Nutr 1999; 69: 250-5.

39. Aghdassi E, Wendiand BE, Steinhart AH, Wolman SL, JeEjeEbHoy K, ALard JP. Antioxidant vitamin supplementation in Crohn's disease decreases oxidative stress. a randomized controlled trial. Am J Gastroenterol 2003; 98: 348-53.

40. Wendiand BE, Aghdassi E, Tam C, Carrrier J, Steinhart AH, Wolman SL et aL. Lipid peroxidation and plasma antioxidant micronutrients in $\mathrm{Crohn}$ disease. Am J Clin Nutr 2001; 74: 259-64.

41. Carrier J, Aghdassi E, Culen J, Aluard JP. Imon supplementation increases disease activity and vitamin $\mathrm{E}$ ameliorates the effect in rats with dextran sulfate sodium-induced colitis. J Nutr 2002; 132: 3146-50.

42. Carrier J, Aghdassi E, Platt I, Culen J, Aluard J. Effect of oral iron supplementation on oxidative stress and colonic inflammation in rats with induced colitis. Aliment Pharmacol Ther 2001; 15: 1989-99.

43. Ademoglu E, Erbil $Y$, Tam B, Barbaros U, Ilhan E, Olgac V et al. Do vitamin E and selenium have beneficial effects on trinitrobenzenesulfonic acidinduced experimental colitis. Dig Dis Sci 2004; 49: 102-8.

44. Loguercio C, D’Argenio G, Delue Cave M, Cosenza V, Dela Vaue N, Mazzacca G et al. Glutathione supplementation improves oxidative damage in experimental colitis. Dig Liver Dis 2003; 35: 63541.

45. Roughead ZK, Johnson LK, Hunt JK. Dietary Copper Primarily Affects Antioxidant Capacity and Dietary Iron Mainly Affects Iron Status in a Surface Response Study of Female Rats Fed Varying Concentrations of Iron, Zinc and Copper micronutrient. J Nutr 1999; 129: 1368-76. 